\title{
Visualizing sustainability in urban conditions
}

\author{
T. Shelton \\ University of Tennessee, TN, USA
}

\begin{abstract}
Cities present many challenges for the environmentally conscious designer. Sustainability is a complex issue comprised of many overlapping and sometimes contradictory concerns. Overlaid on the inherent complexity of the urban environment, questions of sustainability become even more opaque - lost in a multifaceted network well described by Hough. In such conditions it becomes very difficult for even well informed and well intentioned designers to understand the ramifications of their actions.

One of the primary difficulties of sustainable practice in urban conditions is that many of the aspects of urban sustainability are invisible - take for example walkability which may have visible components such as quality of sidewalk or intensity of vehicular traffic but also has many factors that are invisible or not readily seeable such as climate, topography that extends beyond the immediate site, and distribution of amenities. This paper presents research intended to inform designers in the earliest stages of design by making several of these factors visible. Using geographic information visualization (GIV) software multiple aspects of urban sustainability are mapped and visualized. This mapping of previously invisible or partially visible issues in the form of geospatial information provides architects and urban designers with the ability to process and understand information about a particular neighborhood's sustainability through the means with which they are most accustomed - visual information.

As way of example, this research focuses on a particular inner ring neighborhood in Knoxville, Tennessee, USA.
\end{abstract}

Keywords: visualization, mapping, sustainability, urban design. 


\section{Pedagogical context and research structure}

The work was performed in two phases: one supported by an undergraduate research seminar and the other supported by an undergraduate design studio at the University of Tennessee College of Architecture and Design.

\subsection{Phase one}

Phase one was informed by a seminar in the fall semester of 2007 . The seminar began with students familiarizing themselves with the existing conditions of the study area in general terms. This was followed by a directed though wideranging discussion about the meaning of sustainability in urban conditions. This discussion sought to understand and identify the fundamental aspects of urban sustainability and how these might interrelate in North Knoxville. Students were then charged with the following tasks:

Identify particular aspects of urban sustainability on which to focus the study. These identified aspects became the subjects of the various visual information layers developed by the class.

Develop a methodology by which these aspects of sustainability can be measured and ultimately expressed through a visual language.

Analyze the resulting visualizations seeking to understand their implications for neighborhood design.

Recommend targeted design projects as a starting point for the spring semester design studio.

\subsection{Phase two}

Phase two (currently underway) consists of a guided upper level undergraduate research design studio building on the work of the seminar. The studio is charged with:

Suggesting the location and programmatic content of catalytic projects for North Knoxville that will result in improving multiple aspects of urban sustainability identified in the preliminary research.

Providing compelling design proposals incorporating these catalytic projects in the future green development of the area.

Analyzing the effectiveness of their proposals through the lens of the original analyses.

\section{Study area}

North Knoxville is an inner ring neighborhood of Knoxville, Tennessee. Its original lifeblood was a streetcar line that allowed easy access to the center city. As with many American streetcar neighborhoods, North Knoxville possesses many characteristics that make it a strong candidate for reinhabitation and redesign as a green neighborhood - residential areas that are closely aligned with commercial districts, densities that allow for easy bicycle and pedestrian 
mobility, a balance of built area and green space, and proximity to the amenities of downtown, to begin. Hudnut [2].

The designation "North Knoxville" applies to a collection of such neighborhoods. In an effort to be more precise about this research, a boundary was selected that coincided with the recent study performed by the local Metropolitan Planning Commission. This Small Area Plan (SAP), as it is called, focused on the intersection of Central Avenue and Broadway encompassing all or part of many surrounding areas and extending slightly under the interstate toward downtown. Knoxville-Knox County Metropolitan Planning Commission [3]. This boundary describes an area of .45 square miles, fig. 1 .
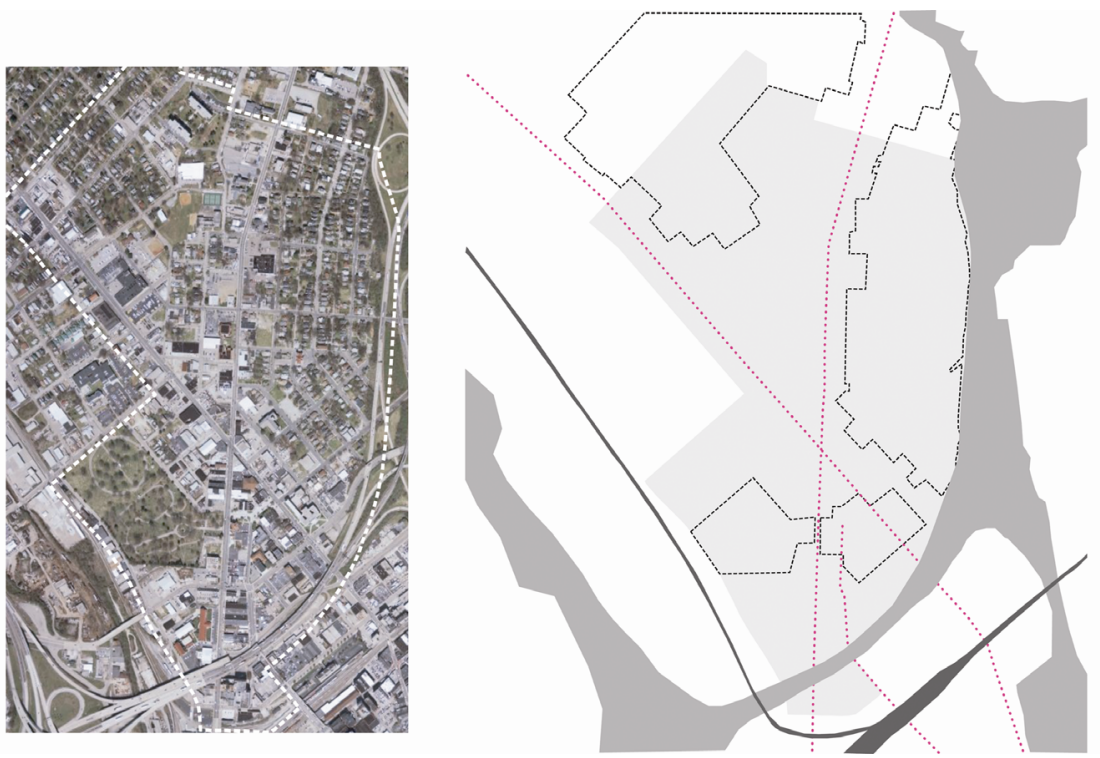

Figure 1: (left) Aerial photograph of North Knoxville with study area boundary indicated. (right) Diagram of study area showing Central Ave. and Broadway, bounding interstates and railroads, and designated historic districts.

\section{Process}

The research seminar identified seven aspects of sustainability to investigate: transit connectivity, stormwater, safety, proximity to amenities, walkability, solar access, and density. The goal of these investigations was to utilize graphic information visualization (GIV) software to display and analyze geospatial visual information related to each of these aspects of urban sustainability. CartaVista ${ }^{\mathrm{TM}}$ GIV software was made available for the work by Ambroziak Third Dimension Technologies, Inc. Some new functions were added to the software during the process to accommodate the class' particular investigations. This software allows for the manipulation of geospatial data in relation to the interests of this study in 
two particular ways. The first involves the creation and manipulation of digital elevation models (DEM) where variables other than elevation are substituted for the $Z$. value. This type of model allows for a wide range of visual outputs and analyses ranging from the application of contour lines and hypsometric tints to the viewing of the models as three dimensional meshes. The second type of manipulation allows for multiple variables to be attached to a single point in space. This allows the possibility of more complex methods of analyzing data that are as yet untapped by this project, including statistical regression analysis.

Over the course of the research four primary methods of visualizing data useful for understanding aspects of urban sustainability have been established cataloging, compilation, coincidence, and interaction.

\subsection{Cataloging}

Cataloging is the simplest of the four methods of visualizing data. It serves two primary purposes - either to reveal simple patterns or holes in patterns or to serve as raw data that will later feed into other more complex analyses. A good example of both of these aspects of cataloging is the tree survey, fig. 2 .

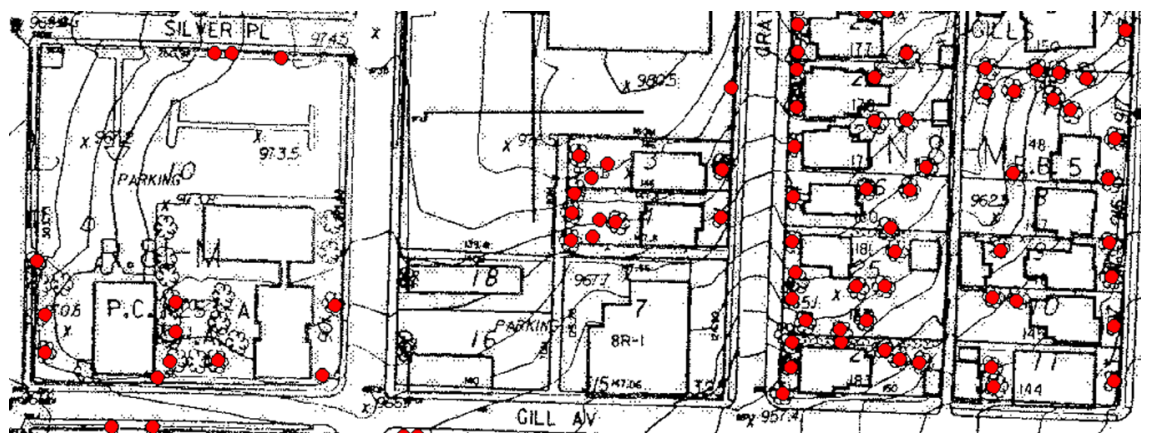

Figure 2: $\quad$ Detail of tree survey.

One can easily analyze the locations of significant trees within the survey area noting particular concentrations or patterns. Such simple straightforward information can be helpful for the design process. However, one of the reasons a tree survey was chosen for the initial cataloging exercise was the potential for information regarding the locations of significant trees to feed into numerous other analyses such as walking comfort, stormwater absorption and evapotranspiration, and solar access. As each point on the tree survey contains information on tree height, canopy diameter, and a classification as to whether each tree is evergreen or deciduous, it is even conceivable that this information could eventually feed into a neighborhood-wide evaluation of carbon balance.

\subsection{Compilation}

Compilation is a more advanced analysis. It involves the combined display of an aspect of sustainability the values of which vary with location. This type of 
investigation presents opportunities to begin to develop visualizations of the unexpected interactions of complex and invisible aspects of urban sustainability.

Walkability provides a strong example of this type of analysis. Through field observation students evaluated the walkability of neighborhood streets, grading them on seven variables ranging from quality of sidewalk surface, to the presence and the nature of buffers between pedestrians and automobile traffic, to shading of the sidewalk. These were compiled into a composite score for each length of sidewalk. This information was then in turn made visual using the GIV software through use of hypsometric tints. The resulting images provide clear information on walkability within the study area that combines a variety of factors and is quickly digested through visual means, fig. 3.
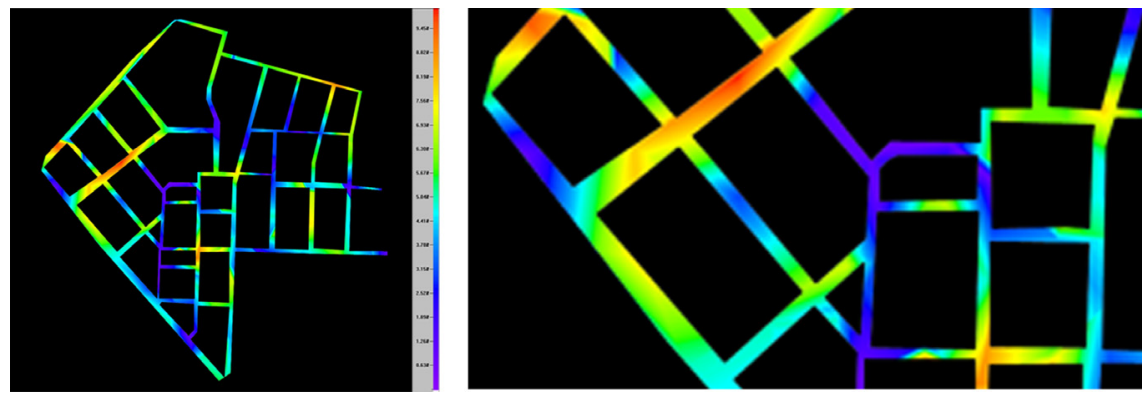

Figure 3: (left) Visual walkability analysis. (right) Detail of walkability analysis.

An example of the importance of both the visual nature of this information and its overt link to location is demonstrated by the stormwater analysis. The stormwater investigation began with a type of cataloging. Working within the watershed defined by the low point at the intersection of Broadway and Central Avenues, students began by dividing each constituent block into 5 foot by 5 foot grids. Each square in the grid was then designated as being predominantly one of five possible surface types: building, pavement, gravel, grass, or trees. Even the resulting pixelated image begins to provide important visual information by calling out large areas of paving within the watershed.

Using standard values for the absorptivity of these various surfaces then allowed the students to predict the amount of stormwater contributed to the city system by each block in a two year storm event (approximately 1.5 inches per hour). Though the seminar students' investigation concluded with extensive and reliable information, its representation in bar graph form renders it very difficult to use during the design process, requiring cross referencing between the graph and a map labelled with the appropriate block numbers. A limited amount of subsequent work with GIV, however, makes the information graphic and links it to location, thus rendering it much more useful for design purposes, fig. 4. 

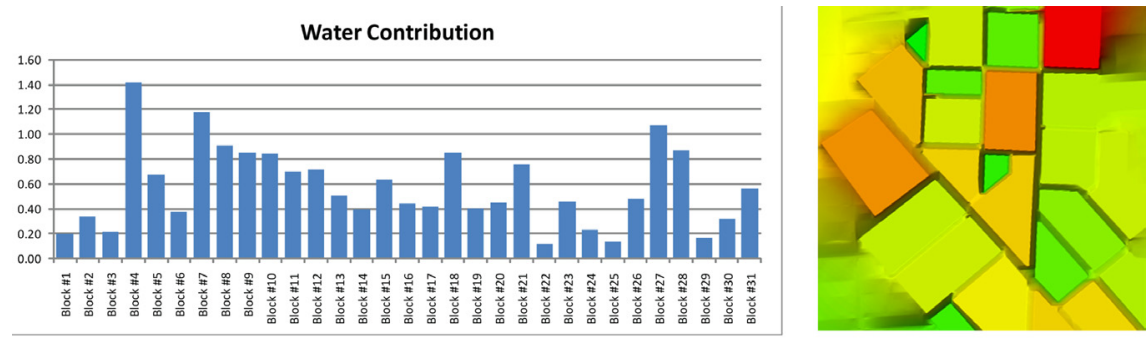

Figure 4: (left) Stormwater contribution as a bar graph and (right) made visual and spatial through GIV (detail of larger map).

\subsection{Coincidence}

Coincidence is a type of analysis where one begins to look at the interactions between related (or seemingly unrelated) aspects of urban sustainability. This type of analysis simply begins to look for an overlapping of these disparate concerns once each has been made visual through either cataloging or compilation. Though the seminar course did not overtly deal with coincidence analysis, participants speculated as to what might be opportune pairings of issues for future investigation. One such example might involve overlaying the stormwater and walkability analyses. If one were to find locations where both walkability was low and the contribution of stormwater to the gutter system was high these areas might then be targeted as locations where a single catalytic project dealing with the street edge might simultaneously improve both aspects of urban sustainability. Coincidence analysis represents the first step toward understanding and acknowledging the interconnectedness of environmental issues and urban issues. As such, it points to new questions and demands design briefs that address several complex issues simultaneously.

\subsection{Interaction}

The most advanced analysis foreseen by this project is interaction. Interaction analysis seeks to combine in one set of data the complex relationships between multiple aspects of urban sustainability, each of which has embedded in it values that vary with location. The true promise of using visual information to detangle and provide a coherent picture of the complex interrelationships between the many aspects of urban sustainability lies in this type of analysis. Aside from the obvious difficulties of compiling the large amounts of information necessary to creating a meaningful interaction analysis, the greatest hurdle is perhaps one of prioritization. An interaction analysis that relates seemingly unrelated aspects of sustainability will require many assumptions as to the relative weight of each. For instance, how does one begin to assign priorities when attempting to combine disparate aspects of urban sustainability such as proximity of housing to transit stops and passive absorption of stormwater? Is one housing unit within 500 feet of a transit stop worth 100 gallons of passive storm water absorption? Is it worth more, less? Although some efforts are underway to quantify and relate 
these issues, ultimately such questions may either need to be tied back to common fundamental measures - carbon, energy, water - or left to individual municipalities as statements of local values, Farr [4].

\section{Detailed compilation analysis: proximity to amenities}

Following the seminar one aspect of urban sustainability has been identified as the subject for a more detailed compilation analysis. This aspect, proximity to amenities, is closely based on a concept found in the pilot version of the LEED $^{\mathrm{TM}}$ for Neighborhood Development rating system - diversity of uses, United States Green Building Council [5]. The diversity of uses concept is straightforward and well-founded. It holds that if commonly used neighborhood amenities are within easy walking distance residents will tend to use more benign modes of travel (walking or bicycling) to access these amenities, thus alleviating some of the demand for car travel. The diversity of uses concept lists nineteen such uses and awards points on a sliding scale based on the number of these located within a $1 / 2$-mile walking distance of the development in question. The compilation analysis examined both the LEED-ND list of uses and an expanded list of uses within and near the study area.

The LEED-ND rating system requires that applicants measure the actual distance of travel when attempting to qualify for these points. This is a distinct improvement over the typical practice of simply drawing a $1 / 2-$ mile radius circle centered on one's development to delineate an easily walkable distance. The LEED method takes into account the effect of obstacles -such as inaccessible topography, super blocks and busy roads - on walking distance. This becomes extremely important when considering walking distance within the urban environment, as is clearly shown by the compilation analysis. The LEED-ND rating system, however, uses an understandable simplification for the purposes of calculating diverse uses points. Every location within the $1 / 2$-mile walking distance is considered to be equal. This sets up a type of binary system where one is either inside or outside of the walking shed - one either receives credit or does not. The diagram below illustrates this simplified understanding of accessibility for three of the uses in the study area: a senior care facility, a fire station, and a restaurant, fig. 5. The small area of the highest saturation indicates where all three of these walking areas overlap and as such is identified as a preferable location even though it is at the outer limits of walking area for two of the uses. However, it is clearly preferable in terms of accessibility to be within $1 / 10$ mile of a given amenity rather than $4 / 10$ mile. While this cannot be taken into account within the limitations of a LEED ${ }^{\mathrm{TM}}$ submittal that already requires a project team to demonstrate performance in many different areas, the subtleties of this type of gradation of benefit is exactly the type of information that is most easily grasped through visual analysis.

For this analysis CartaVista ${ }^{\mathrm{TM}}$ was used to generate digital elevation models. Each amenity was taken as a type of $100 \%$ location where the most desirable position in terms of access to that amenity is immediately adjacent to it. From there, radiating contours of walking distances were drawn in $1 / 10$ mile 

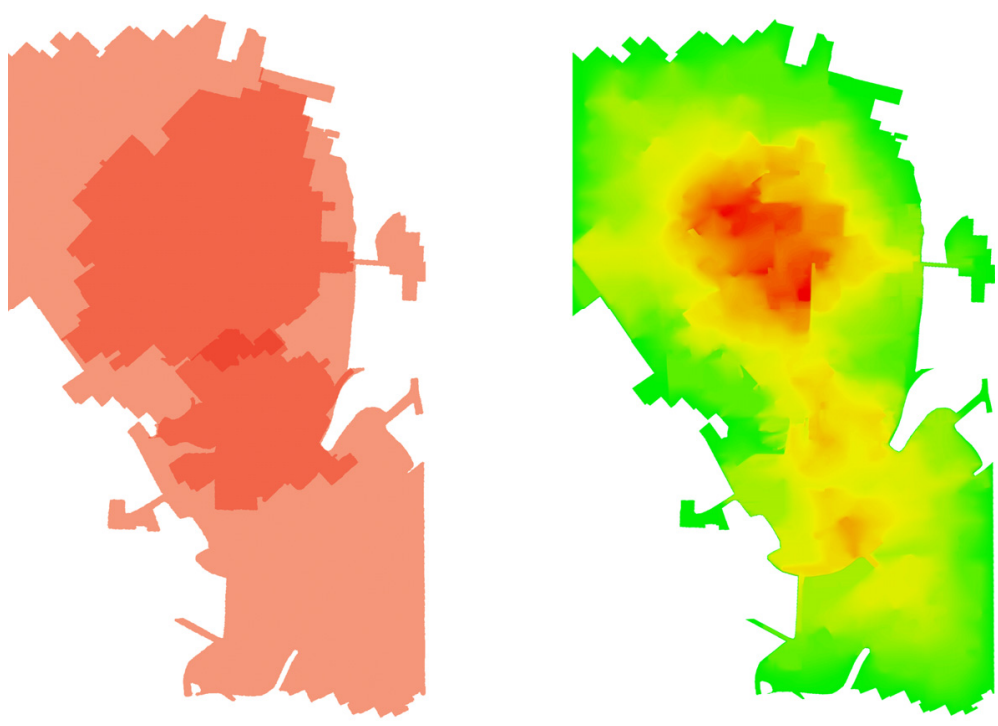

Figure 5: (left) Proximity to three neighborhood amenities understood through the lens of the LEED-ND rating system. (right) Compilation analysis of the same.

increments until $1 / 2$-mile from the amenity had been achieved. Each of these contour lines was then assigned a point value in two point increments with the line immediately adjacent to the amenity receiving ten points and the outermost line receiving zero. Ambroziak Third Dimension Technologies added a function to CartaVista ${ }^{\mathrm{TM}}$ that allowed for multiple digital elevation models to be added to each other for the purposes of this investigation. Using this function we are able to create a composite image of accessibility to amenities across the study area. This diagram shows proximity to the same three amenities, though now clearly accounting for the importance of distance, fig. 5 .

To date thirty-five neighborhood amenities have been mapped and added to the proximity to amenities compilation analysis, fig. 6 . Through these images one begins to understand the profound isolation of the study area and the impact of Broadway and Central Avenues, which can only be traversed at particular locations. These obstacles clearly begin to contain accessibility to amenities located in between the two. The influence of super blocks such as the Guy B. Love Towers in the north and the Old Gray Cemetery in the south are clearly depicted as holes in the accessibility. It is further surprising to see how quickly proximity to amenities drops off within the Fourth and Gill neighborhood (at eastern edge of study area) which ostensibly seems quite well connected.

\section{Conclusion}

This project is but a step toward being able to understand the interactions of environmental concerns in urban conditions through visual means. Though 

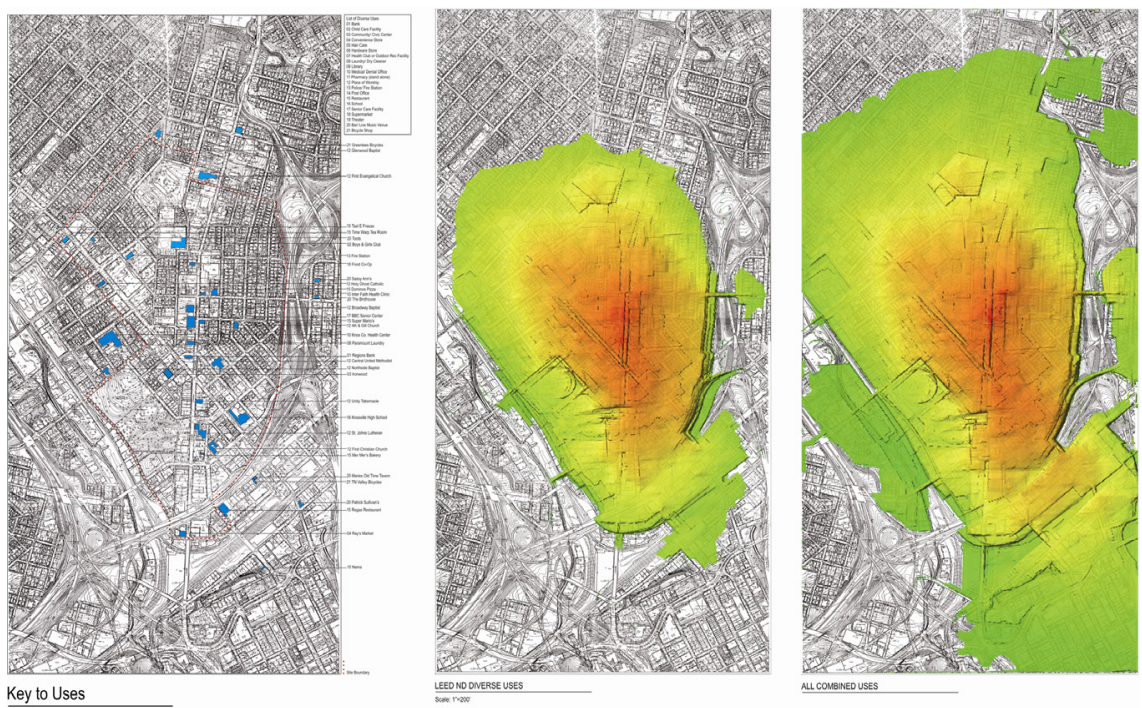

Figure 6: (left) Location of amenities in compilation analysis. (center) Analysis of uses on LEED list. (right) Analysis of uses on extended list.

comprehensive images of the multiple aspects of urban sustainability will require significantly more effort, this project has laid the groundwork for such investigations by both demonstrating the usefulness of the information embodied in the constituent analyses and developing a clearer understanding of methods by which these might be combined into more complex information models.

This analysis recognizes urban entities - neighborhoods, districts, cities - as intricately functioning organisms comprised of many interrelated systems. As such, an analogy can be made between the potential usefulness of this effort and the role visual information plays in medicine. If architects, planners, and urban designers were to consider the sustainability of a neighborhood as something akin to its health, then interventions in that neighborhood could be focused, adjusted, and targeted toward its improvement. Visualizations of the various aspects of urban sustainability might then become as invaluable to the work of the designers as X-rays, sonograms, and MRIs are to physicians.

\section{Acknowledgements}

The following organizations have provided financial and material support for this project: AIA Board Knowledge Committee, AIA East Tennessee Chapter, the University of Tennessee College of Architecture and Design, and Ambroziak Third Dimension Technologies Incorporated. Of particular assistance were Brian Ambroziak and Russell Ambroziak. Their work in both explaining and extending the functionality of the CartaVista ${ }^{\mathrm{TM}}$ software was invaluable. 


\section{References}

[1] Hough, Michael, Cities and Natural Process: a Basis for Sustainability, Routledge: London and New York, pp. 19-25, 1995.

[2] Hudnut III, William H., Halfway to Everywhere: A Portrait of America's First-Tier Suburbs, Urban Land Institute: Washington, pp. 417-420, 2003.

[3] Knoxville-Knox County Metropolitan Planning Commission, BroadwayCentral-Emory Place Small Area Plan, Knoxville, TN, http://archive.knoxmpc.org/plans/smallarea/becp_March2007.pdf

[4] Farr, Douglas, Sustainable Urbanism: Urban Design with Nature, Wiley: Hoboken, pp. 54-55, 2008.

[5] United States Green Building Council, LEED for Neighborhood Development Rating System, Pilot Version, Washington, http://www.usgbc.org/ShowFile.aspx?DocumentID=2845 